\title{
Ältere Ein- und Zweifamilienhausbestände im Umbruch. Eine Clusteranalyse zur Identifizierung regionaler Betroffenheiten in Nordrhein-Westfalen
}

\author{
Brigitte Adam $^{1} \cdot$ Andrea Berndgen-Kaiser $^{2} \cdot$ Kerstin Jochimsen $^{3} \cdot$ Angelika Münter $^{4}\left(\mathbb{D} \cdot\right.$ Philipp Zakrzewski $^{5}$
}

Eingegangen: 27. Juni 2016 / Angenommen: 12. Januar 2017 / Online publiziert: 26. Januar 2017

(c) Springer-Verlag Berlin Heidelberg 2017

Zusammenfassung Im Zusammenspiel von demographischen Faktoren, Merkmalen des Wohnungsangebotes, raumstrukturellen Faktoren und der daraus resultierenden Gesamtmarktsituation trifft vielerorts im Segment der Einund Zweifamilienhäuser (EZFH) der 1950er- bis 1970erJahre eine sinkende Nachfrage auf ein wachsendes Angebot an Bestandsgebäuden. Die regionale Betroffenheit der Gemeinden ist jedoch sehr unterschiedlich. Im vorliegenden Beitrag werden am Beispiel Nordrhein-Westfalens ein Indikatorenkonzept zur Abschätzung der Lage der Bestände an Ein- und Zweifamilienhäusern der 1950er- bis 1970er-

Dr. Brigitte Adam

brigitte.adam@bbr.bund.de

Andrea Berndgen-Kaiser

andrea.berndgen-kaiser@ils-forschung.de

Kerstin Jochimsen

kerstin.jochimsen@nrwbank.de

$\triangle$ Dr. Angelika Münter

angelika.muenter@ils-forschung.de

Philipp Zakrzewski

philipp.zakrzewski@mlv.sachsen-anhalt.de

1 Bundesinstitut für Bau-, Stadt-, und Raumforschung (BBSR) im Bundesamt für Bauwesen und Raumordnung, Deichmanns Aue 31-37, 53179 Bonn, Deutschland

2 ILS - Institut für Landes- und Stadtentwicklungsforschung, Büro Aachen, Karmeliterstraße 6, 52064 Aachen, Deutschland

3 NRW.BANK, Friedrichstraße 1, 48145 Münster, Deutschland

4 ILS - Institut für Landes- und Stadtentwicklungsforschung, Brüderweg 22-24, 44135 Dortmund, Deutschland

5 Ministerium für Landesentwicklung und Verkehr des Landes Sachsen-Anhalt, Turmschanzenstraße 30, 39114 Magdeburg, Deutschland
Jahre dargestellt und einzelne Indikatoren hinsichtlich regionaler Muster ausgewertet. Der Fokus des Beitrages liegt auf der anschließenden Synthese der Indikatoren mittels einer Clusteranalyse (Two-Step-Verfahren). Im Ergebnis werden sechs Gemeindecluster mit ähnlichen Entwicklungen und Herausforderungen bezüglich der Bestände an Ein- und Zweifamilienhäusern der 1950er- bis 1970er-Jahre identifiziert und die spezifischen Handlungserfordernisse in den Clustern diskutiert. Aus der methodischen Perspektive verdeutlicht der Beitrag, dass eine Clusteranalyse nach dem Two-Step-Verfahren eine vielversprechende Alternative zum häufig in den Raumwissenschaften angewendeten Ward-Verfahren sein kann.

Schlüsselwörter Einfamilienhausgebiete - Demographie · Wohnungsmarkt · Raumstruktur - Indikatoren ·

Clusteranalyse

\section{Elderly Single-Family Homes in Transition. A Cluster Analysis to Identify Regional Challenges in North Rhine-Westphalia}

Abstract Within the interplay of demographic factors, characteristics of the housing stock, spatial parameters and the resulting conditions on the housing market in many municipalities a declining demand for single family homes built in the 1950s to 1970s goes along with an increasing supply in this segment of the housing market. At this, however, strong regional disparities can be observed. In this paper, a concept of indicators on the municipal level to estimate the situation of the 1950s to the 1970s single-family homes is presented. Based on the example of North RhineWestphalia in a first step regional spatial patterns are identified based on some of these indicators. The focus of the 
paper is on the subsequent synthesis of the indicators by means of a cluster analysis (two-step method). As a result, six clusters with similar developments and challenges regarding the 1950 s to 1970 s single-family-homes stocks are identified and the operative requirements for these clusters are discussed. From the methodological perspective the paper shows that the cluster analysis by the means of the twostep method is a promising alternative to the Ward method frequently adopted in spatial sciences.

Keywords Single-family homes - Demography · Housing market $\cdot$ Spatial patterns $\cdot$ Indicators $\cdot$ Cluster analyses

\section{Einleitung}

Ein- und Zweifamilienhausbestände der 1950er- bis 1970erJahre befinden sich gegenwärtig in einer Phase des Umbruchs. Zur Zeit ihrer Erbauung wurden diese Gebiete von jungen Familien bezogen, sodass sie heute zunächst einer kollektiven Alterung ihrer Bewohner und anschließend einem Generationenwechsel unterliegen. Da gleichzeitig die Zahl der neu gegründeten Familienhaushalte zurückgeht, trifft zunehmend eine sinkende Nachfrage auf ein wachsendes Angebot von Bestandsgebäuden. Dies geschieht aufgrund eines Auseinanderdriftens der Bevölkerungsentwicklung Nordrhein-Westfalens in Wachstums- und eine zunehmende Zahl von Schrumpfungsregionen (vgl. IT.NRW 2015), jedoch in regional höchst unterschiedlichem Maße. Viele Einfamilienhäuser dieser Baujahre erfüllen zudem häufig nicht mehr die heutigen Wohnanforderungen bezüglich Größe, Zuschnitt und energetischer Qualität.

Obwohl der Markterfolg der Wohnform „Einfamilienhaus"1 dadurch nicht grundsätzlich in Frage gestellt wird, ist jedoch $\mathrm{zu}$ erwarten, dass eine Ausdifferenzierung in unterschiedlich erfolgreiche Teilbestände hinsichtlich der jeweiligen Objekteigenschaften und der groß- und kleinräumigen Lage erfolgen wird (Wüstenrot-Stiftung 2012; Adam/Berndgen-Kaiser/Jochimsen et al. 2015). Dies für Nordrhein-Westfalen zu überprüfen, ist Ziel dieses Beitrages. Insbesondere soll die Bandbreite unterschiedlicher Betroffenheiten, also möglicherweise problematischer Entwicklungen und Herausforderungen in den Ein- und Zweifamilienhausbeständen der 1950er- bis 1970er-Jahre auf Gemeindeebene mithilfe einer Clusteranalyse herausgearbeitet werden. Im folgenden Kapitel wird zunächst der

\footnotetext{
1 Aus einer inhaltlichen Perspektive nimmt dieser Beitrag die Wohnform „Einfamilienhaus“ in den Blick, die neben freistehenden Häusern auch Doppel- und Reihenhäuser umfasst. Die statistische Annäherung hieran sind „Wohnungen in Wohngebäuden mit ein bis zwei Wohneinheiten“, hier als „Ein- und Zweifamilienhäuser“ abgekürzt. Diese Kennziffer umfasst freistehende Einfamilienhäuser sowie Doppel- und Reihenhäuser mit bis zu zwei Wohneinheiten.
}

Stand der Forschung zur Lage und zum Umgang mit diesen Beständen dargelegt. In Kapitel 3 wird darauf aufbauend ein Indikatorenkonzept zur Abschätzung der Lage von Einund Zweifamilienhausbeständen der 1950er- bis 1970erJahre entwickelt und in Kapitel 4 werden ausgewählte Indikatoren visualisiert. Anschließend werden die Ergebnisse in Kapitel 5 mittels einer Clusteranalyse zu einer Typologie verdichtet und in Kapitel 6 die spezifischen Handlungsbedarfe für die einzelnen Cluster hinsichtlich des planerischen Umgangs mit älteren Ein- und Zweifamilienhausbeständen benannt. Kapitel 7 zieht ein inhaltliches sowie ein methodisches Fazit und arbeitet den weiteren Forschungsbedarf heraus.

\section{Stand der Forschung zur Lage und zum Umgang mit Ein- und Zweifamilienhausbeständen der 1950er- bis 1970er-Jahre}

Europaweit lebten $201459 \%$ der Bevölkerung in Ein- und Zweifamilienhäusern (Eurostat 2016). Obwohl das Einfamilienhaus immer noch vielen Menschen als Wohnideal gilt, mehren sich die Indizien dafür, dass diese Bestände unter Druck geraten. Durch den demographischen Wandel, die Veränderungen der Nutzerpräferenzen, aber auch infolge der Wirtschafts- und Finanzkrisen drohen stagnierende oder nachgebende Preise, Veräußerungsschwierigkeiten und teils sogar Leerstände (Cunningham-Sabot/Fol 2009; Sousa/Pinho 2013; Wiechmann/Bontje 2013; IÖR 2015; Haase/Bernt/Großmann et al. 2016). Diese soziodemographischen und wirtschaftlichen Rahmenbedingungen sind im europäischen Kontext in unterschiedlichen Ausprägungen $\mathrm{zu}$ beobachten, die Forschung $\mathrm{zu}$ älteren Einfamilienhausbeständen steckt jedoch noch in den Anfängen (Berndgen-Kaiser/Fox-Kämper/Wiechert 2016). Lediglich für die Situation in Deutschland liegen erste Erkenntnisse zu den (zukünftigen) Herausforderungen älterer Ein- und Zweifamilienhausbestände vor (Dransfeld/ Pfeiffer/Platzek 2009; Wüstenrot-Stiftung 2012; Reicher/ Hoffschröer/Mecklenbrauck et al. 2013; Berndgen-Kaiser/ Bläser/Fox-Kämper et al. 2014; Bayerische Staatsregierung 2015).

Wie in den meisten Industrienationen befindet sich die Einwohnerzahl Deutschlands - trotz derzeit hoher Zuwanderungen - auf einem generellen Schrumpfungspfad und ab etwa 2020 wird auch die Zahl der Haushalte stagnieren (Statistisches Bundesamt 2016). Diese Entwicklungen gehen mit einer zunehmenden regionalen Polarisierung einher, z. B. zwischen wirtschaftsstarken und strukturschwachen sowie urbanen und ländlichen Regionen. Die Auswirkungen des demographischen Wandels auf die Wohnungsmärkte werden bereits seit Langem beschrieben (Martinez-Fernandez/Audirac/Fol et al. 2012; Lerbs 2014; Held/ 
Waltersbacher 2015). Mit einem heutigen Bestand von circa 15 Millionen Ein- und Zweifamilienhäusern und einem Anteil am Wohnungsbestand in den Kommunen von bis zu $80 \%$ (je nach Lage und Zentralität) prägt dieser Gebäudetyp die Siedlungsstruktur Deutschlands in besonderem Maße (Berndgen-Kaiser/Krajewski 2015: 171). Angesichts der weiterhin vorhandenen Wertschätzung gering verdichteter Wohnformen und des im internationalen Vergleich eher geringen Anteils von Ein- und Zweifamilienhäusern am Wohnungsbestand wurde lange Zeit davon ausgegangen, dass diese Bestände von einer rückläufigen Wohnungsnachfrage kaum betroffen sein würden. Für die Kommunen waren Einfamilienhausgebiete nach der Erschließung und Bebauung oft ,Selbstläufer', sie bedurften kaum weiterer Steuerung und waren gleichzeitig Garant für Bevölkerungswachstum und Steuereinnahmen (Aring 2012).

In den letzten Jahren rückt jedoch zunehmend die Frage nach der Zukunft älterer Einfamilienhausgebiete in den Fokus (Rosenbohm 2005; Zakrzewski 2011; Wüstenrot-Stiftung 2012; Berndgen-Kaiser/Bläser/Fox-Kämper et al. 2014; von Seckendorf/Müller-Herbers/Skorka 2014; StMUV/StMAS/StMELF et al. 2014; Bayerische Staatsregierung 2015; Müller-Herbers/Skorka 2016). Diese Diskussion wird vor allem von drei Überlegungen bestimmt.

Erstens wird der Einfluss des demographischen Wandels auf die zukünftige Nachfrage nach Einfamilienhäusern diskutiert. Die Bestandseigenheime wurden von Angehörigen relativ stark besetzter Bevölkerungskohorten gebaut und werden heute überwiegend noch von ihnen bewohnt. Da die nachfolgenden Generationen potenzieller Erwerber jedoch zunehmend geburtenschwachen Jahrgängen angehören, ergeben sich quantitative Diskrepanzen, wenn eine wachsende Menge frei werdender Häuser auf eine abnehmende Zahl nachfragender Haushalte trifft (de Temple 2006; Dransfeld/ Pfeiffer/Platzek 2009; Zakrzewski 2011).

Zweitens können gesellschaftliche Veränderungen wie die Pluralisierung der Lebensstile, der Bedeutungsverlust traditioneller Familienmodelle (Fina/Planinsek/Zakrzewski 2010), der Anstieg der Frauenerwerbsquote und die Erosion des Normalarbeitsverhältnisses zu einem generellen Attraktivitätsverlust der Wohnform Eigenheim und somit zu qualitativen Diskrepanzen führen (Dransfeld/Pfeiffer/ Platzek 2009; Zakrzewski 2011). Neue Erwerber sehen ihr Einfamilienhaus zudem häufig nicht mehr als ,Lebenswerk', sondern als lebensabschnittsorientierte Wohnform. Ohne Anpassungen werden besonders ältere Einfamilienhäuser diesen veränderten Lebensweisen und Ansprüchen nicht genügen (Berndgen-Kaiser/Krajewski 2015). In vielen Bestandsgebieten wird der erforderliche Bewohnerund Generationswechsel nur dann erfolgreich verlaufen, wenn die homogenen Strukturen aufgebrochen werden können, indem Haushalte unterschiedlicher Größe sowie
Alters und Lebensstils der Haushaltsmitglieder als neue Nachfragergruppen auftreten (Hohn/Utku 2015).

Die dritte Herausforderung für den Fortbestand der Gebiete bezieht sich auf räumliche Polarisierungen zwischen Wachstums- und Schrumpfungsregionen und zentralen und peripheren Standorten. Demnach ist bei älteren Einfamilienhausgebieten in zentralen und randstädtischen Lagen von prosperierenden Regionen mit langfristig wachsender Nachfrage und steigenden Preisen zu rechnen. Dagegen können Eigenheime an peripheren Standorten in Schrumpfungsregionen mit Nachfrageproblemen und Wertverfall konfrontiert sein (Aehnelt/Winkler-Kühlken 2008; Hahne 2010; Spehl 2011). Das bedeutet, dass in Deutschland in einigen Regionen weiterhin eine Unterversorgung im Segment der Ein- und Zweifamilienhäuser besteht, während andere Gebiete auf ein Überangebot zusteuern. Diese räumliche Polarisierung macht sich schon seit Längerem in der Wertentwicklung von älteren Ein- und Zweifamilienhausbeständen in Deutschland bemerkbar (Aehnelt/WinklerKühlken 2008; Maßß 2015).

Die aktuelle Situation der Zuwanderung von Flüchtlingen nach Deutschland führt kurzfristig zu einem eklatanten Mangel an Wohnungen. Ob und welche Einflüsse dies auf die Marktgängigkeit älterer Ein- und Zweifamilienhausbestände haben wird, kann derzeit noch nicht abschließend abgeschätzt werden. Allerdings sprechen die Erfahrungen aus vergangenen Zuwanderungswellen dafür, dass insbesondere die Ballungsräume mit ihren bereits heute höheren Anteilen an Migranten und damit auch besseren Netzwerken sowie ihren häufig besseren Erwerbschancen das dauerhafte Ziel von anerkannten Flüchtlingen sein werden (o. V. 2015; Postlep 2015). Die aktuelle Zuwanderungswelle wird damit mutmaßlich einerseits demographisch bedingte Schrumpfungsprozesse - auch in peripheren Räumen - für einige Jahre dämpfen, aber gleichzeitig regionale Disparitäten eher weiter verstärken als zu ihrem Ausgleich beitragen.

Über diese generellen Erkenntnisse zur Zukunft älterer Ein- und Zweifamilienhausbestände hinaus verdeutlichen empirische Fallstudien zu einzelnen Regionen, dass die Bandbreite der Entwicklungsperspektiven dieser Bestände in ,problematischen ' Regionen von Preisanpassungen über den Markt bis hin zu Verödung und Verfall ganzer Quartiere reicht. Für Nordrhein-Westfalen wird konstatiert, dass sich die Marktchancen der Bestände nicht nur in Abhängigkeit von der großräumigen Lage, sondern auch der kleinräumigen Lage sowie der Gebäudequalität ausdifferenzieren werden. Kleinräumig befinden sich die Bestandsimmobilien der 1950er-Jahre meist in den besten Lagen, während die ,Verlierer' peripher gelegene Bestände minderer bis mittlerer Qualität sein werden, zu denen häufig in den 1960er-/1970er-Jahren erbaute Bestände zählen (Dransfeld/ Pfeiffer/Platzek 2009: 49 f.). Für Hessen, Rheinland-Pfalz und das Saarland wird außerdem betont, dass problema- 
tische Konstellationen nicht nur großräumig in entlegenen Schrumpfungsregionen, sondern auch kleinräumig in einem stagnierenden oder wachsenden Umfeld auftreten können (Spellerberg/Spehl 2011: 1). Standl (2006: 297) erwartet für periphere Gemeinden in Bayern zukünftig sogar ,postmoderne Wüstungserscheinungen“. Heute organisiert sich der Umbruch der Gebiete in der Regel mit Preisanpassungen und der Erschließung preissensibler Nachfragegruppen über den Markt. Hier bleibt allerdings unklar, ob diese Marktbalance auch mittelfristig beibehalten werden kann (Aring 2015). Denn in besonders stark schrumpfenden Städten, wie Altena, zeigen sich in peripher gelegenen Beständen bereits heute strukturelle Leerstände und es ist zu erwarten, dass diese zukünftig zunehmen werden (Krajewski 2015).

Auf dieser empirischen Problembeschreibung aufbauend legen einige teils umfangreiche Studien mit unterschiedlichen disziplinären Zugängen und Untersuchungsschwerpunkten einen Fokus auf Handlungsoptionen (Schöfl/ Schluchter/Schöfl et al. 2005; Aehnelt/Winkler-Kühlken 2008; Wüstenrot-Stiftung 2012; Bayerische Staatsregierung 2015). So stellt beispielsweise die „Wüstenrot-Studie“ einen umfangreichen, Werkzeugkasten ' an Instrumenten für die Kommunen zur Sicherung dieser Bestände zusammen. Die Handlungsoptionen reichen von einer sorgfältigen Erfassung und Analyse der Gebiete über eine bestandsorientierte Siedlungsentwicklung und Aktivierung von Baulücken und Brachen bis zu interkommunalen Abstimmungsprozessen (Wüstenrot-Stiftung 2012).

Dieser Beitrag widmet sich einem spezifischen Ausschnitt aus der hier skizzierten Problematik. Ziel ist es, die Bandbreite unterschiedlicher Betroffenheiten, also möglicherweise problematischer Entwicklungen und Herausforderungen in den Ein- und Zweifamilienhausbeständen der 1950er- bis 1970er-Jahre auf der Gemeindeebene in Nordrhein-Westfalen aufzuzeigen. Die Gemeinden sollen mittels einer Clusteranalyse zu einer Typologie verdichtet werden, die Gemeindetypen umfasst, welche quantitative, qualitative und räumliche Unterschiede hinsichtlich der Marktfähigkeit dieser Wohnungsbestände aufweisen. Nur so können auch planerische Handlungsbedarfe regional differenziert benannt werden. Neben der fachspezifischen Dimension spielt der methodische Ansatz gleichermaßen eine Rolle. Mit ihm - also mit dem Vorgehen bei der Clusterung - soll ein Exempel geliefert werden, wie mit komplexen Indikatorenkonzepten bei einer Vielzahl an Fällen (hier: Gemeinden) verfahren werden kann.

\section{Indikatorenkonzept anhand von vier Themenbereichen}

Wie im vorangegangenen Kapitel dargelegt, werden Probleme im Marktsegment der Ein- und Zweifamilienhäuser der 1950er- bis 1970er-Jahre vor allem dann erwartet, wenn das Angebot der auf den Markt gelangenden Bestandsein- und -zweifamilienhäuser die demographisch bedingte Nachfrage nach diesem Objekttyp übersteigt oder wenn ungenügende Lage- und Qualitätsmerkmale der Gebäudebestände einen Wiederverkauf erschweren. Unterschiedliche regionale Betroffenheiten entstehen also durch ein Zusammenspiel von vier Komponenten bzw. Themenbereichen:

- demographische bzw. nachfragebedingte Faktoren am Wohnungsmarkt,

- Angebotsmerkmale des Wohnungsmarktes,

- raumstrukturelle Faktoren bzw. Lageeigenschaften sowie

- die aus den ersten drei Komponenten resultierende Gesamtsituation am Wohnungsmarkt, also vor allem die Preise.

Diese vier Themenbereiche dienen im Folgenden als Grundgerüst für die Abschätzung regionaler Betroffenheiten hinsichtlich der Marktfähigkeit und der Entwicklungsperspektiven älterer Ein- und Zweifamilienhäuser. Zunächst werden Leitfragen formuliert, welche die Themenfelder anhand dieser Zielsetzung charakterisieren. Diese werden anschließend in Indikatoren ,übersetzt ${ }^{*}$ (vgl. Tabelle 1). Da die Analysen auf der Gemeindeebene durchgeführt werden, werden nur Indikatoren berücksichtigt, die auf dieser Ebene auch verfügbar, valide und reliabel sind (vgl. Adam/Berndgen-Kaiser/Jochimsen et al. 2015). Strukturdaten werden je nach Datenverfügbarkeit für die Jahre 2011 (Zensus) oder 2012 dargestellt, Entwicklungsdaten in der Regel für den Fünfjahreszeitraum 2007 bis 2012. Um eine gleichmäßige Gewichtung der vier Blöcke in der Clusteranalyse zu gewährleisten (vgl. Kapitel 5), wird für jeden Block eine gleiche Anzahl an Indikatoren berücksichtigt. Mit drei Indikatoren je Block gelingt es, die Leitfragen zu allen Blöcken angemessen abzudecken (vgl. Tabelle 1). ${ }^{2}$

Demographie: Die Veränderungen der Nachfrage am Wohnungsmarkt im Allgemeinen und speziell nach älteren Ein- und Zweifamilienhäusern wird im Wesentlichen durch die demographische Entwicklung bestimmt und zwar sowohl durch die Bevölkerungsentwicklung insgesamt schrumpft oder wächst eine Gemeinde? - als auch durch Veränderungen der Altersstruktur. Beide Faktoren sind maßgeblich dafür, in welchem Umfang und mit welcher Dynamik zukünftig Bestandsimmobilien, die heute von älteren Menschen bewohnt werden, neu auf dem Wohnungsmarkt angeboten werden. Obwohl der Beitrag darauf

\footnotetext{
${ }_{2}$ Die Leitfragen und die Auswahl der Indikatoren wurden nicht nur im Kreis der Autoren dieses Beitrages, sondern darüber hinaus intensiv in Sitzungen der Arbeitsgruppe „Einfamilienhausgebiete der 50er und 60er-Jahre" der Landesarbeitsgemeinschaft Nordrhein-Westfalen der Akademie für Raumforschung und Landesplanung diskutiert. Für eine ausführliche Begründung des Indikatorenkonzeptes vgl. Adam/ Berndgen-Kaiser/Jochimsen et al. (2015).
} 
Tabelle 1 Leitfragen und Indikatoren zur Abschätzung unterschiedlicher Betroffenheiten in den Ein- und Zweifamilienhausbeständen der 1950er- bis 1970er-Jahre

Themenbereiche und Leitfragen
Demographie
- Wächst oder schrumpft die Bevölkerung und wie
verändert sich die Altersstruktur?
- Welche demographisch bedingten Umbrïche am
Wohnungsmarkt sind zu erwarten?

Wohnungsangebot

- Welche Bedeutung hat der Bestand an Einfamilienhäusern der 1950er- bis 1970er-Jahre innerhalb des Wohnungsbestandes?

- Gibt es (bereits) Leerstände im

Einfamilienhaussegment?

\author{
Indikatoren \\ für die allgemeine demographische Entwicklung die Bevölkerungsentwicklung von \\ 2007 bis 2012 in \% \\ für das zu erwartende Freiwerden an Wohnraum die durchschnittliche Zahl der Ster- \\ befälle 2011-2029 je 1.000 Einwohner 2011 \\ als Proxyindikator für den Anteil von Familien als potenziellen neuen Nachfragern der \\ Anteil der unter 18-Jährigen an der Gesamtbevölkerung 2012
}

\section{Raumstruktur}

- Ist die Region durch eine hohe oder geringe Bevölkerungsdichte geprägt?

- Wie gut oder schlecht ist es um die Erreichbarkeit von Einrichtungen der Daseinsvorsorge bestellt?

\section{Wohnungsmarkt}

- Wie stellen sich Niveau und Entwicklung der Boden- und Immobilienpreise dar?

- Über welche Kaufkraft verfügt die Bevölkerung? für den Bestand an älteren Ein- und Zweifamilienhäusern der Anteil der Wohneinheiten in Ein- und Zweifamilienhäusern an allen Wohneinheiten der Baujahre 19491978

für den Leerstand in dieser Baualtersklasse der Anteil leer stehender Wohneinheiten an allen Wohneinheiten in Ein- und Zweifamilienhäusern der Baujahre 1949-1978 für eine Einschätzung der Reaktion auf aktuelle Trends im Neubau die Veränderung des Baufertigstellungsniveaus zwischen 2003 und 2007 und 2008 bis 2012 in Wohneinheiten je 1.000 Einwohner

für die Dichte der Besiedlung die Einwohnerdichte 2012 in Einwohner/km²

für die Zentralität und Anbindung an öffentliche Verkehrsmittel die durchschnittliche Erreichbarkeit des nächsten Oberzentrums von allen Haltestellen einer Gemeinde in Minuten

für die Nahversorgung die durchschnittliche Luftliniendistanz zum nächsten Supermarkt/Discounter in $\mathbf{m}$

für die private Finanzkraft die Kaufkraft je Einwohner 2011 in $€$

für das generelle Preisniveau die Baulandpreise 2012 in $€ / \mathbf{m}^{2}$

für die Wertstabilität von Wohnimmobilien die Preisentwicklung der Ein- und Zweifamilienhäuser im Bestand 2006-2010 abzielt, zukünftige Herausforderungen der älteren Ein- und Zweifamilienhäuser am Markt zu identifizieren, werden für die Analyse der demographischen Effekte zwei strukturelle bzw. rückblickende Indikatoren verwendet. Denn Bevölkerungsvorausberechnungen sind auf der kleinräumigen Ebene der Gemeinden mit sehr großen Unsicherheiten insbesondere bei den Wanderungsbewegungen behaftet (vgl. Gatzweiler 1996). Für das zu erwartende Freiwerden an Wohnraum aus dem Bestand kann hingegen ein prognostischer Indikator (Sterbefälle 2011-2029) genutzt werden. Denn für diesen sind kaum Unsicherheiten hinsichtlich der Wanderungsannahmen zu erwarten, da er überwiegend die älteren Alterskohorten umfasst, deren Wanderungsneigung im Allgemeinen sehr gering ist (BIB 2015).

Wohnungsangebot: Aktuell bereits vorhandene Angebotsüberhänge spiegeln sich in einer erhöhten Leerstandsquote wider. Leerstandsquoten bis $3 \%$ gelten als notwendige Fluktuationsreserve, um Wohnungswechsel von Bewohnern und Renovierungsarbeiten $\mathrm{zu}$ ermöglichen, Werte darüber zeigen strukturelle Leerstände an (Brack/ Metzmacher 2009). Die Anpassungsbedarfe im Marktsegment der älteren Ein- und Zweifamilienhäuser sind umso größer, je bedeutender diese Bestände am Gesamtmarkt sind. Wird gleichzeitig der Gesamtwohnungsbestand durch
Neubau beständig vergrößert, steigt die Gefahr zukünftiger Angebotsüberhänge.

Raumstruktur: Auf den Immobilienmärkten beeinflussen groß- und kleinräumige Lageeigenschaften maßgeblich die Preisgestaltung. Aus der Sicht der räumlichen Planung sind raumstrukturelle Merkmale vor allem wegen ihres unmittelbaren Einflusses auf die Lebensqualität bedeutend. Wie weit ist es zu Schulen, Einkaufsmöglichkeiten oder Ärzten? Und wie erreiche ich die nächste große Stadt? Die Einwohnerdichte verweist auf die theoretischen Möglichkeiten zur Ausstattung der Gemeinde mit Angeboten der sozialen Infrastruktur und des öffentlichen Nahverkehrs. Einkaufsmöglichkeiten (täglicher Bedarf) vor Ort erhöhen die Gunst einer Gemeinde als Wohnstandort. Fehlende Einrichtungen vor Ort können daher den Generationswechsel erschweren. Die Erreichbarkeit von Oberzentren mit dem öffentlichen Verkehr gibt hingegen Hinweise auf großräumige Erreichbarkeiten und die Lageattraktivität einer Gemeinde.

Wohnungsmarkt: Ob und inwieweit die demographisch determinierte Wohnungsnachfrage am Markt mit einem entsprechenden Angebot befriedigt werden kann, kommt durch die Preise und deren Entwicklung sowie die finanziellen Möglichkeiten der Haushalte, als Nachfrager von Wohneigentum aufzutreten, zum Ausdruck. Der Strukturindikator 
Baulandpreisniveau verweist auf Lageeigenschaften sowie darauf, ob die Nachfrage höher ausfällt als das Angebot oder umgekehrt. Niedrige Baulandpreise geben außerdem einen Hinweis darauf, dass der Wohnungsneubau unter Umständen genauso günstig ist wie der Erwerb und die Modernisierung einer Bestandsimmobilie (Ziegler 2013). Als Indikator zur Preisentwicklung wurde hingegen bewusst ein Indikator gewählt, der sich nur auf den Bestand bezieht, um eine nachlassende Nachfrage nach diesem Marktsegment gezielt abbilden zu können.

\section{Räumliche Muster ausgewählter Indikatoren der vier Themenbereiche}

Bevor die zwölf Indikatoren im nächsten Abschnitt zu einer Cluster-Typologie verdichtet werden, sollen in diesem Abschnitt zunächst die räumlichen Muster in den vier Themenbereichen visualisiert werden. Dies dient im Sinne eines analytischen Zwischenergebnisses dazu, einen mehrdimensionalen Überblick über unterschiedliche Aspekte der Marktfähigkeit der älteren Ein- und Zweifamilienhausbestände zu geben. Für jeden der vier Themenbereiche wird dabei derjenige Indikator herausgegriffen, der die Leitfragen zum jeweiligen Themenfeld am besten zusammenfasst. ${ }^{3}$

Die räumlichen Muster für diese Indikatoren (vgl. Abbildung 1) lassen sich wie folgt skizzieren:

Demographie (Indikator: relative Bevölkerungsentwicklung 2007-2012): Die demographische Entwicklung in Nordrhein-Westfalen war in den letzten Jahren durch eine Reurbanisierung unter Schrumpfungsbedingungen gekennzeichnet. Die höchsten Bevölkerungsverluste waren dabei in den ländlich geprägten Räumen entlang der östlichen Landesgrenze zu beobachten.

Wohnungsangebot (Indikator: Anteil der Wohneinheiten in Ein- und Zweifamilienhäusern an allen Wohneinheiten der Baujahre 1949-1978): $18 \%$ aller Wohnungen bzw. $43 \%$ aller Wohnungen in Ein- und Zweifamilienhäusern in Nordrhein-Westfalen sind Ein- und Zweifamilienhäuser der Baualtersklasse 1949-1978. Die räumliche Verteilung zeigt insbesondere ein Land-Stadt-Gefälle.

Raumstruktur (Indikator: Luftliniendistanz zum nächsten Supermarkt/Discounter): Innerhalb Nordrhein-Westfalens zeigt sich nicht nur ein Stadt-Land-Gefälle hinsichtlich der Versorgung mit Einkaufsmöglichkeiten, sondern es zeigen sich auch kleinräumige Disparitäten (z. B. innerhalb des suburbanen Raumes).

Wohnungsmarktsituation (Indikator: Baulandpreisniveau 2012 in Euro je $\mathrm{m}^{2}$ ): Die durchschnittlichen Baulandpreise in Nordrhein-Westfalen sind insbesondere durch ein Stadt-

\footnotetext{
${ }^{3}$ Eine ausführliche Interpretation aller Indikatoren findet sich in Adam/Berndgen-Kaiser/Jochimsen et al. (2015).
}

Land-Gefälle mit einer sehr großen Spannweite gekennzeichnet. Regional betrachtet sind die niedrigsten Preise in Ost- und Südwestfalen sowie der Nordeifel zu beobachten, also jenen Regionen, in denen auch die höchsten Bevölkerungsverluste festzustellen sind.

Im Vergleich der vier Indikatoren lassen sich zwei für die Marktfähigkeit älterer Ein- und Zweifamilienhausbestände relevante regionale Muster identifizieren: Zum einen zeigt sich ein Stadt-Land-Gefälle entlang der Typologie der Großstadtregionen des Bundesinstituts für Bau, Stadtund Raumforschung. ${ }^{4}$ Je ländlicher die Raumkategorie, desto eher deuten die Indikatoren auf eine Betroffenheit hin. Zum anderen werden unterschiedliche Muster im Hinblick auf die demographischen Entwicklung deutlich, die sich nur zum Teil mit dem beschriebenen Stadt-Land-Gefälle decken. Diese beiden Grundmuster überlagern sich in der Leerstandsquote älterer Ein- und Zweifamilienhäuser (vgl. Abbildung 2). Abbildung 2 verdeutlicht gleichzeitig, dass aktuell Wohnungsleerstände in diesem Marktsegment in Nordrhein-Westfalen nur ein punktuelles Problem sind - nur elf Kommunen weisen bisher moderat erhöhte Leerstände von mehr als $5 \%$ (bis maximal $7 \%$ ) auf. Betroffenheit von Umbrüchen in älteren Ein- und Zweifamilienhausbeständen ist in Nordrhein-Westfalen daher (bisher) nicht mit Leerstand gleichzusetzen, sondern vielmehr ein mehrdimensionales und sich voraussichtlich in den nächsten Jahren verstärkendes Phänomen (vgl. Kapitel 2). Mit der im Folgenden dargestellten Cluster-Typologie sollen daher vor allem die regionalen Unterschiede der zukünftigen Marktfähigkeit dieser Bestände und der planerischen Handlungserfordernisse im Umgang mit diesen herausgestellt werden.

\section{Clusterung der Gemeinden mit vergleichbaren Herausforderungen in den Ein- und Zweifamilienhausbeständen der 1950er- bis 1970er-Jahre}

Problem- und handlungsrelevante Ausprägungen der Indikatoren aus den vier Themenbereichen sollen im letzten Analyseschritt zur Einschätzung der Lage von Ein- und Zweifamilienhausbeständen der 1950er- bis 1970er-Jahre systematisch zur Synopse gebracht werden. Hierzu werden die Gemeinden anhand der Indikatoren klassifiziert und zu Gruppen (Clustern) zusammengeführt, die ähnliche Entwicklungsbedingungen und Herausforderungen aufweisen. Diese Klassifizierung erlaubt es, die 396 Gemeinden als Einzelfälle zu wenigen Gruppen so zuzuordnen, dass strukturelle Problemlagen und Handlungsoptionen eindeutiger

\footnotetext{
${ }^{4}$ Vgl. http://www.bbsr.bund.de/BBSR/DE/Raumbeobachtung/ Raumabgrenzungen/Grossstadtregionen/Grossstadtregionen.html? $\mathrm{nn}=443048$ (21.11.2016).
} 


\section{A) Bevölkerungsentwicklung 2007-2012}

\section{in Prozent}

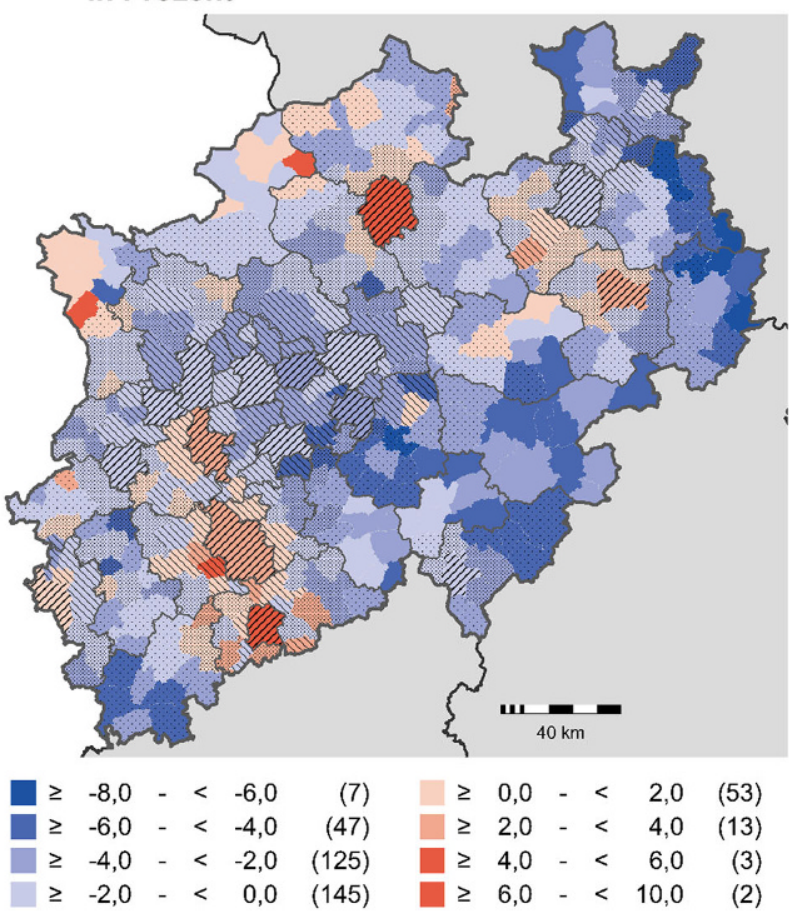

C) Baulandpreise 2012 in Euro je $\mathrm{m}^{2}$

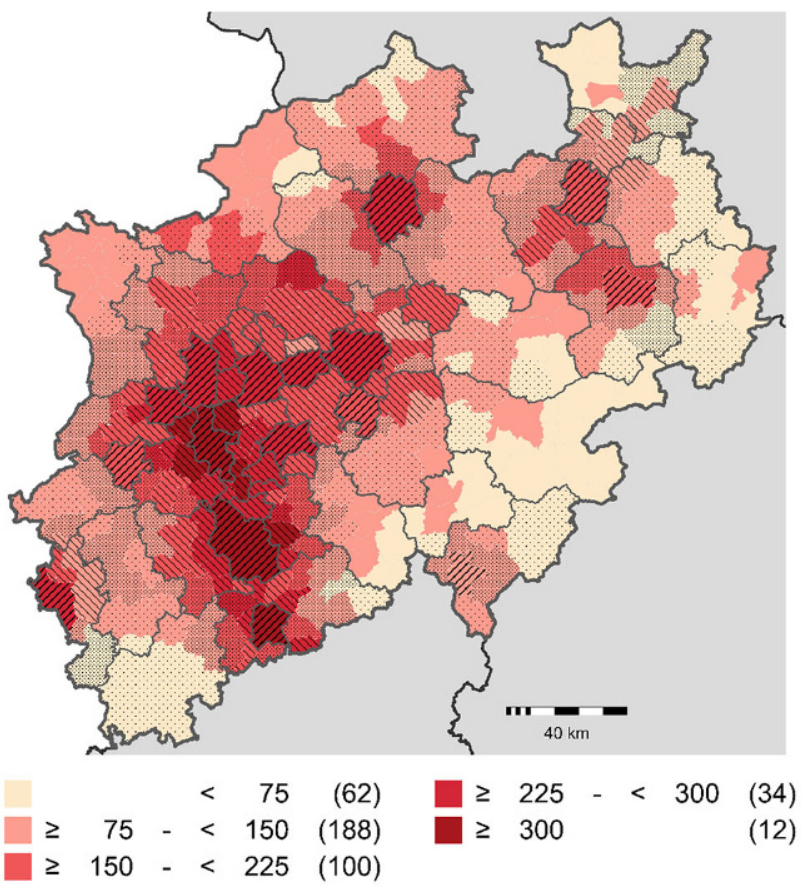

Legende Karten A)-D)

BBSR-Großstadtregionen
B) Anteil Wohnungen in EZFH der Baujahre

1949-1978 am Gesamtwohnungsbestand

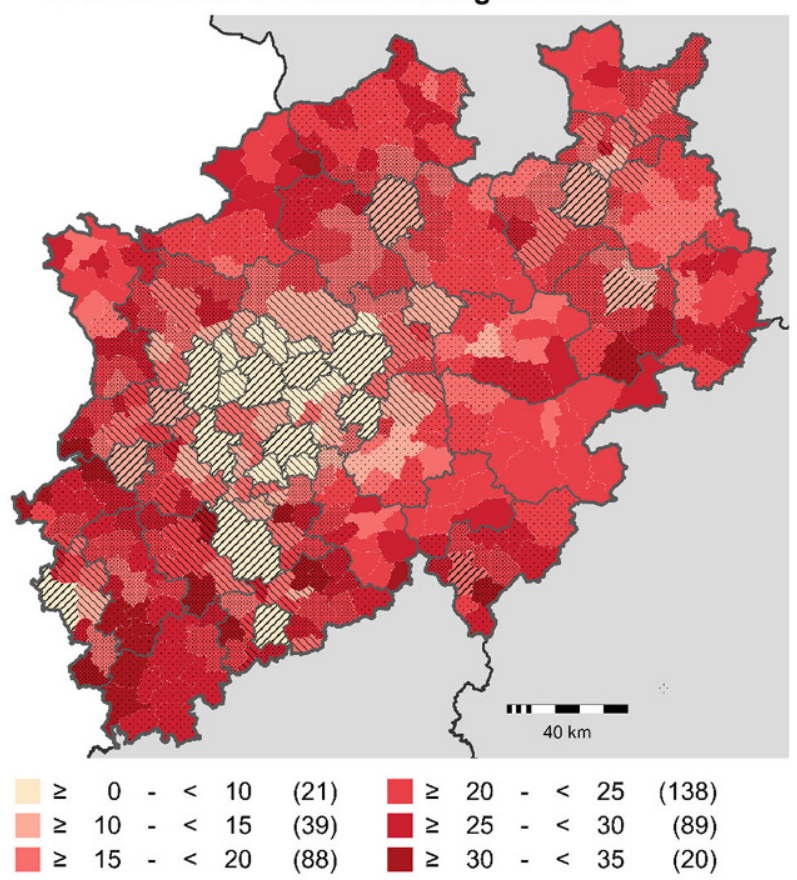

D) Erreichbarkeit von Supermärkten und

Discountern (Luftliniendistanz in Metern)

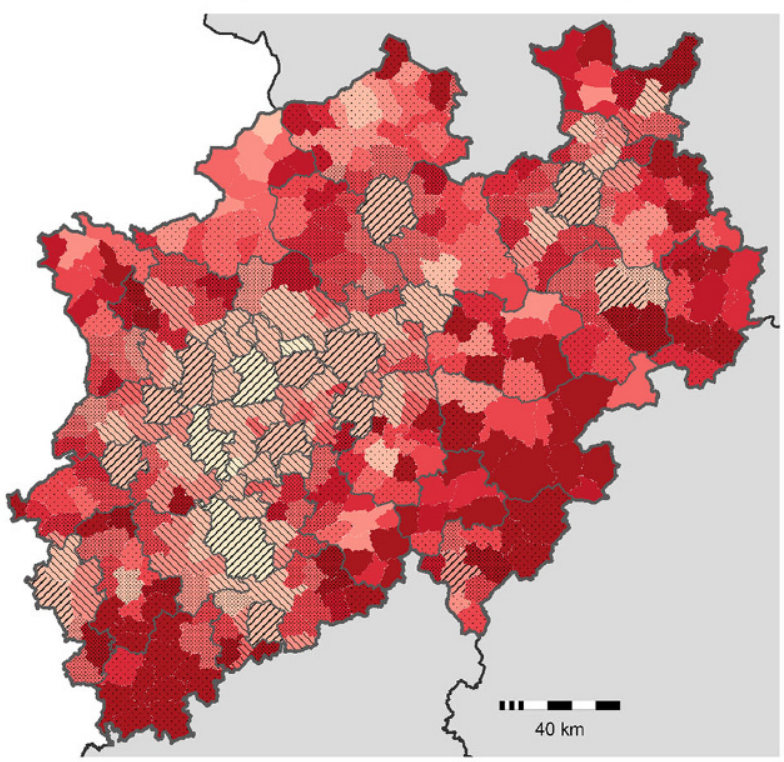

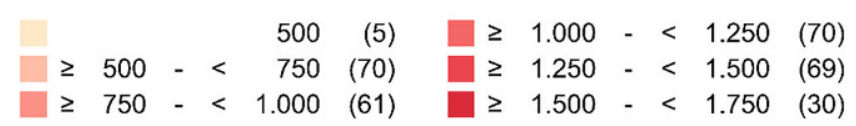

Kern È Ergänzungsgebiet zum Kern engerer Pendlereinzugsbereich

weiterer Pendlereinzugsbereich $\square$ außerhalb von Stadtregionen

Abbildung 1 Räumliche Muster ausgewählter Indikatoren zur Einschätzung der Lage von Ein- und Zweifamilienhausbeständen der 1950er- bis 1970er-Jahre in Nordrhein-Westfalen Quelle: verändert aus Adam/Berndgen-Kaiser/Jochimsen et al. (2015: 179 ff.); Datengrundlagen: A) IT.NRW (Bevölkerungsfortschreibung auf der Basis der Volkszählung 1987; B) Zensus 2011; C) Laufende Raumbeobachtung des Bundesinstituts für Bau-, Stadt- und Raumforschung; D) Oberer Gutachterausschuss Nordrhein-Westfalen 
Abbildung 2 Leerstandsquote (2011) in Wohneinheiten in Ein- und Zweifamilienhäusern der Baualtersklasse 1949-1978 Quelle: verändert nach Adam/ Berndgen-Kaiser/Jochimsen et al. (2015: 180); Datengrundlage: Gebäude- und Wohnungszählung 2011 (Sonderauswertung)

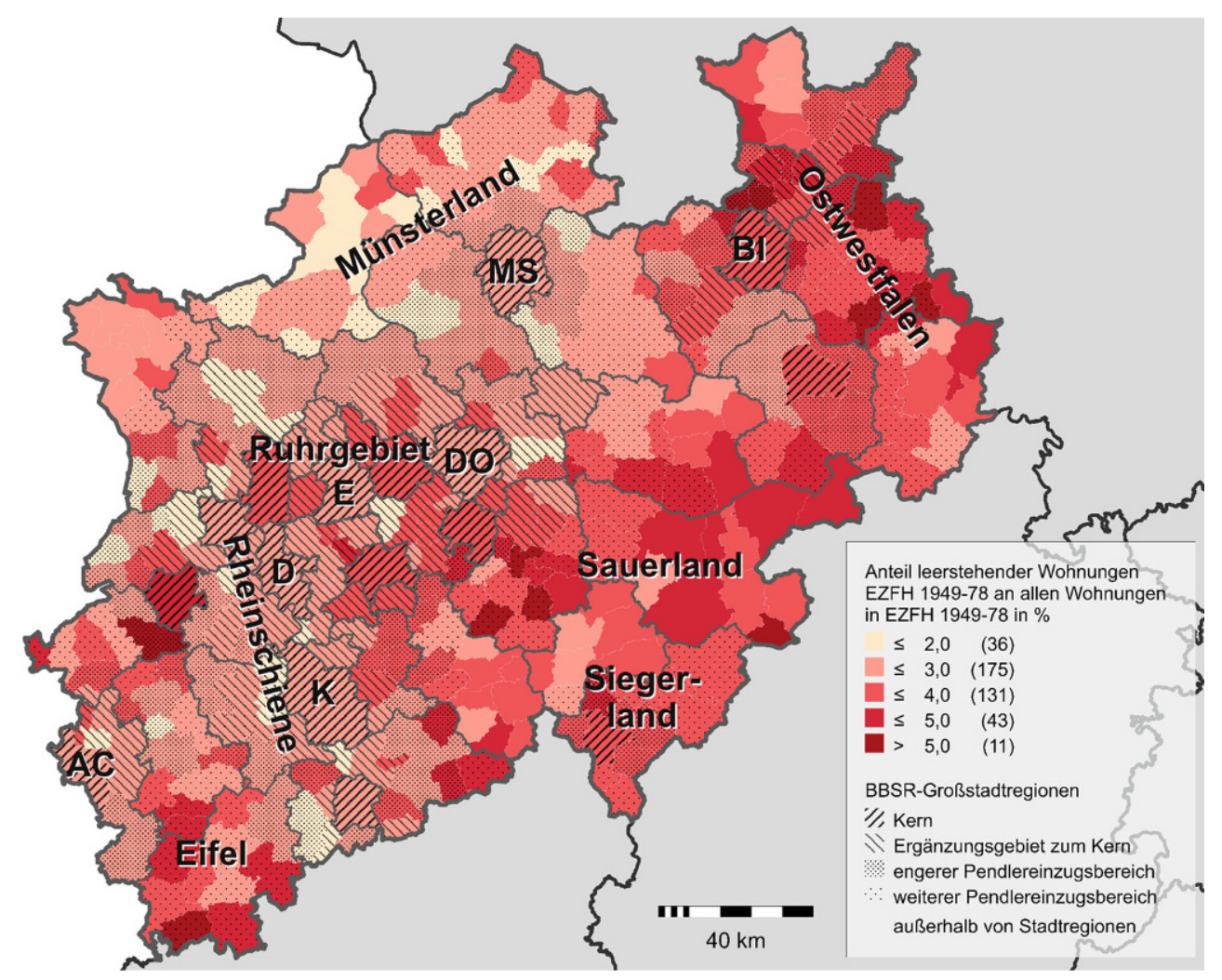

und übergeordnet abgeleitet werden können (vgl. Bahrenberg/Giese/Mevenkamp et al. 2008: 259) als durch die Visualisierung der einzelnen Indikatoren. Da hinsichtlich der Ein- und Zweifamilienhausbestände der 1950er- bis 1970erJahre bisher keine Raumtypen entwickelt worden sind und somit keine Vorkenntnisse für eine geeignete Regionalisierung der nordrhein-westfälischen Gemeinden zu dieser Fragestellung vorliegen, bietet sich die Clusteranalyse als statistisches Verfahren zur Gruppenbildung an. Als exploratives Verfahren kann diese eingesetzt werden, um noch unbekannte Strukturen in den Daten zu entdecken (Bahrenberg/Giese/Mevenkamp et al. 2008: 287).

\subsection{Methodik der Clusteranalyse}

Grundsätzlich stehen verschiedene statistische Verfahren zur Gruppenbildung (Clusterung) zur Verfügung. Welches Verfahren mit welchen methodischen Spezifikationen jeweils gewählt wird, hängt davon $\mathrm{ab}$, wie die Indikatorenwerte beschaffen sind, welche Abhängigkeiten die Indikatoren untereinander aufweisen, wie hoch die Zahl der Objekte ist und ob zugrunde liegende Hypothesen möglicherweise eine bestimmte Anzahl der zu unterscheidenden Gruppen vorgeben (Backhaus/Erichson/Plinke et al. 2016: $449 \mathrm{ff}$.).

Unter den verschiedenen Clusterverfahren fiel die Entscheidung auf die „Two-Step-Methode“. Dieses Verfahren entfaltet bei unserem Analysezweck gegenüber den klassischen hierarchischen Verfahren (z. B. Single-Linkage, Complete-Linkage, Ward) sowie gegenüber den partitionierenden Verfahren (z. B. k-Means-Verfahren) (vgl. Bacher 1996) zwei für den vorliegenden Datensatz relevante Vorteile. Erstens erlaubt die Methode eine höhere Flexibilität durch die zweistufige Zuordnung. Zunächst werden sogenannte Clusterbäume erzeugt (stufenweises Vorgehen der Gruppenbildung), anschließend können bereits zugeordnete Objekte (Gemeinden) im Laufe des Verfahrens die Gruppe wechseln, was im Ergebnis zu einer besseren $\mathrm{Zu}$ ordnung der untersuchten Gemeinden führen sollte (Hoops/ Schäfer 2008: 18). Insbesondere vor dem Hintergrund der relativ hohen Fallzahl von 396 Objekten ist eine solche iterative Herangehensweise geboten. Zweitens gilt dieses Verfahren als robust gegen eine Annahmeverletzung der Normalverteilung, der Unabhängigkeit und der Topologie der Variablen (Hoops/Schäfer 2008: 18; IBM SPSS 2012: 503). Nur die Hälfte der zwölf Indikatoren ist normalverteilt. Der Indikator Einwohnerdichte wird wegen der hohen Korrelation zu den Indikatoren Anteil der Wohnungen in Ein- und Zweifamilienhäusern der Baualtersklasse 1949-1978 und Baulandpreisniveau 2012 ausgeschlossen. Die verbleibenden Indikatoren weisen trotz geringer bis mäßiger bivariater Korrelationen ein Kaiser-Meyer-OlkinKriterium von 0,707 auf. Es kann also davon ausgegangen werden, dass die Variablen nicht gänzlich unabhängig 
voneinander sind (Backhaus/Erichson/Plinke et al. 2016: $336 \mathrm{ff}$.)

Gemäß des Kaiser-Meyer-Olkin-Kriteriums erscheint es sinnvoll, der Cluster- eine Faktorenanalyse voranzuschalten. Die Faktorenanalyse führte zu vier Faktoren, die sich in ähnlicher Weise zusammensetzten, wie sie bereits aus inhaltlichen Überlegungen in den vier Themenblöcken zusammengefasst wurden (vgl. Kapitel 3). Nur die Indikatoren der Themenblöcke Wohnungsangebot und Wohnungsmarkt wurden in zwei Faktoren gemischt und zwei Indikatoren einem anderen Faktor zugeordnet. Da nicht alle Indikatoren die erforderliche Normalverteilung aufweisen, ist das Ergebnis der Faktorenanalyse mit Bedacht zu interpretieren. Wegen der Annahmeverletzung und des wenig vom theoretischen Gerüst der vier Themenbereiche abweichenden Ergebnisses, wurde die Clusteranalyse letztendlich nicht auf Basis der Faktoren, sondern unmittelbar auf der Grundlage der verbliebenen elf Indikatoren durchgeführt. Alle Indikatoren sind metrisch skaliert. In diesem Falle wäre die Euklidische Distanz das geeignete Distanzmaß für die Analyse (IBM SPSS 2012: 504). Dieses Distanzmaß führte aber im konkreten Fall zur Bildung von zwei kleinsten Gruppen mit jeweils zwei Gemeinden und einer Gruppe von 352 Gemeinden. Das Ergebnis überzeugte insofern kaum, da erstens eine Gruppe fast alle Gemeinden Nordrhein-Westfalens umfasst. Zudem erschließt sich unter Berücksichtigung der Mittelwerte und der Streuung die Besonderheit der beiden nur doppelt besetzten Gruppen nicht. Sie repräsentieren keine Ausreißer - auch nicht in einzelnen Variablen. Daher wurde die Clusteranalyse mit dem Likelihood-Maß durchgeführt, welches eine gleichmäßigere Gruppenverteilung erzeugt.

\subsection{Ergebnisse mittels „Two-Step-Methode“}

Es ergibt sich eine optimale Clusterzahl, die zwischen vier und acht Clustern liegt. Alle Varianten führen zu fast gleichen Ergebnissen beim Silhouetten-Kohäsionstest mit durchschnittlich 0,2. Dies entspricht einer mäßigen Verteilung der Werte zum jeweiligen Clustermittelpunkt. Über die anschließende Bewertung der Cluster mittels deskriptiver Analyse erwiesen sich sechs Cluster als besonders sinnvoll und gut interpretierbar sowie gleichzeitig maximal unterscheidbar (vgl. Abbildung 3 und Tabelle 2).

Über die Clusterung der elf Indikatoren werden im Wesentlichen die großräumigen Lageeigenschaften weiter nach Problemkategorien der demographischen Entwicklung und des Marktes für Ein- und Zweifamilienhäuser differenziert. In zwei Clustern finden sich Gemeinden, die schlecht an die Oberzentren und an Einrichtungen der Nahversorgung angebunden sind. Cluster 1 (,periphere schrumpfende Kommunen im ländlichen Raum") umfasst Gemeinden mit einem hohen Anteil an älteren Ein- und
Zweifamilienhäusern, mit den deutlichsten Bevölkerungsrückgängen, dem geringsten Baulandpreisniveau sowie dem deutlichsten Preisrückgang im Bestand. Auch die Leerstandsquote älterer Ein- und Zweifamilienhäuser ist höher als in den übrigen Clustern, allerdings mit 3,65\% auch in diesem Cluster (bisher) im Mittel nur leicht über der Fluktuationsreserve. Es handelt sich um Gemeinden in der Nordeifel, dem Sauerland sowie im östlichen Ostwestfalen. Das zweite Cluster peripher gelegener Gemeinden (Cluster 2 ,periphere stabile Kommunen im ländlichen Raum“) zeigt in Bezug auf die raumstrukturellen Indikatoren sowie das Baulandpreisniveau Ähnlichkeiten zum Cluster 1, allerdings auf einem höheren Niveau. Der sehr hohe Anteil an Familien bei gleichzeitig positiver Preisentwicklung im Bestand deutet auf einen funktionierenden Generationenwechsel hin. $\mathrm{Zu}$ diesem sehr kleinen Cluster zählen vor allem Gemeinden im Münsterland außerhalb des engeren Verflechtungsbereiches von Münster sowie südwestlich von Paderborn.

Zwei weitere Cluster umfassen kleinere und mittlere Städte ohne Erreichbarkeitsdefizite. Sie unterscheiden sich vornehmlich durch die demographische Entwicklung. Im Cluster 3 (,stabile Klein- und Mittelstädte") ist die Bevölkerungszahl annähernd stabil und weist einen überdurchschnittlichen Anteil an Kindern und Jugendlichen auf. Es handelt sich hierbei um suburbane und ländliche Gemeinden im Münsterland, das suburbane Umland Bielefelds sowie kleinere Städte und Gemeinden im Rheinland und an der niederländischen Grenze. Die Gemeinden des Clusters 4 (,schrumpfende Kommunen ohne Erreichbarkeitsdefizite") haben dagegen einen überdurchschnittlichen Bevölkerungsverlust zu verzeichnen, die Leerstandsquote ist leicht über- und das Baulandpreisniveau unterdurchschnittlich. Die Gemeinden dieses Clusters grenzen überwiegend entweder an schrumpfende periphere Gemeinden (Cluster 1) oder an schrumpfende urbane Gemeinden des Clusters 6 an, z. B. in den Randbereichen des Ruhrgebietes, in Ostwestfalen oder im Siegerland.

Bei den Clustern 5 und 6 handelt es sich um urbane Gemeinden mit einem vergleichsweise geringen Anteil an älteren Ein- und Zweifamilienhäusern am Wohnungsbestand. Beide unterscheiden sich aber ganz deutlich in der demographischen Entwicklung und in den Effekten auf dem Markt für (ältere) Ein- und Zweifamilienhäuser. Im Cluster 5 (,wachsende Großstädte und deren suburbanes Umland") vereinen sich die hochpreisigen Gemeinden mit demographisch überwiegend positiven Entwicklungen. In diesem Cluster finden sich die Kernstädte der Rheinschiene und ihr hochverdichtetes Umland sowie weitere wirtschaftlich prosperierende Großstädte (z. B. die Universitätsstädte Bielefeld und Münster). Die urbanen Kommunen des Clusters 6 (,altindustrialisierte Großstädte und deren suburbanes Umland (stagnierend bis schrumpfend)“) befinden sich 


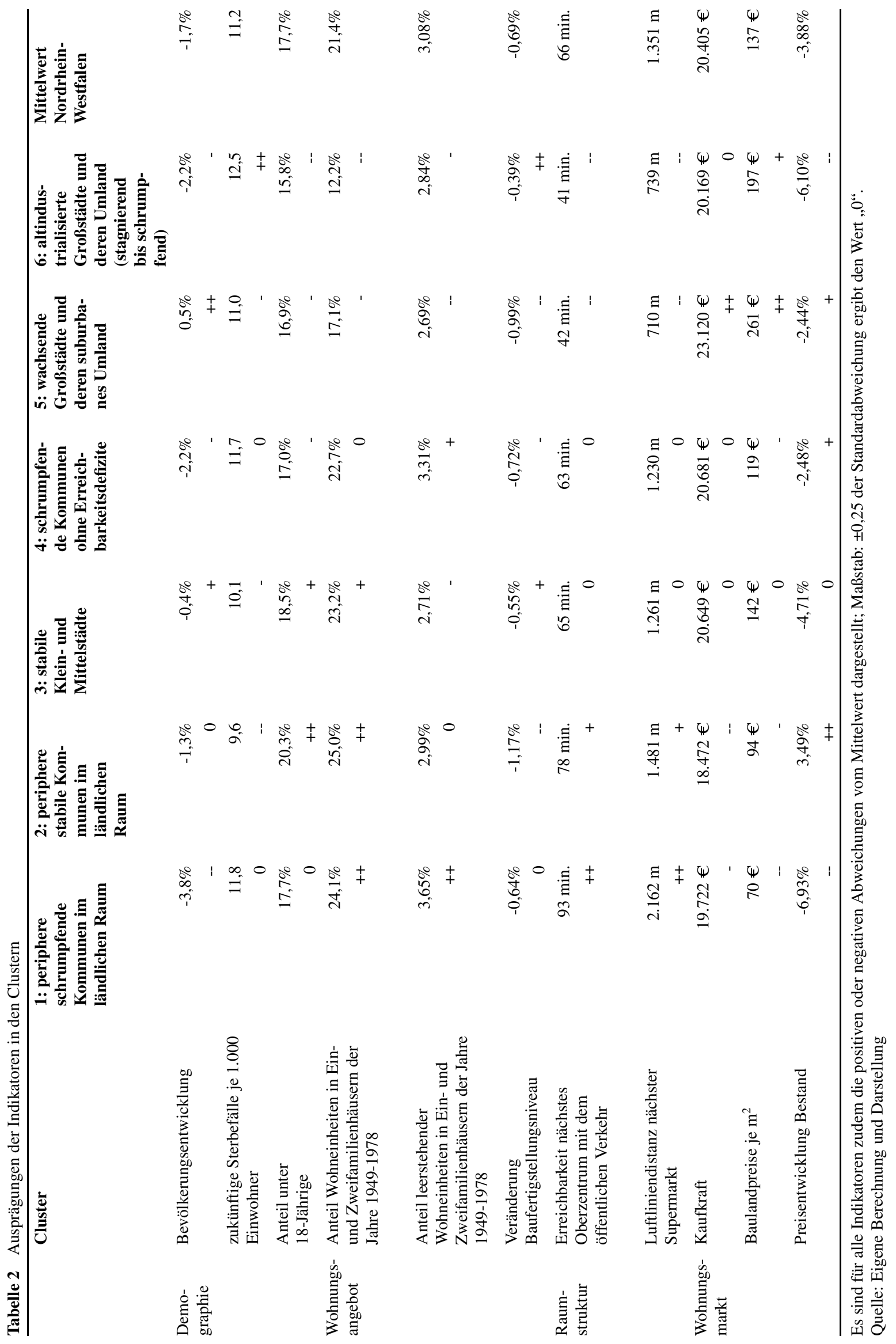


Abbildung 3 Gemeindecluster zur Einschätzung der Lage von Ein- und Zweifamilienhausbeständen der 1950er- bis 1970erJahre in Nordrhein-Westfalen Quelle: eigene Berechnung und Darstellung; geometrische Grundlage: BKG, Gemeinden 31.12.2013; Bearbeitung: A. Milbert

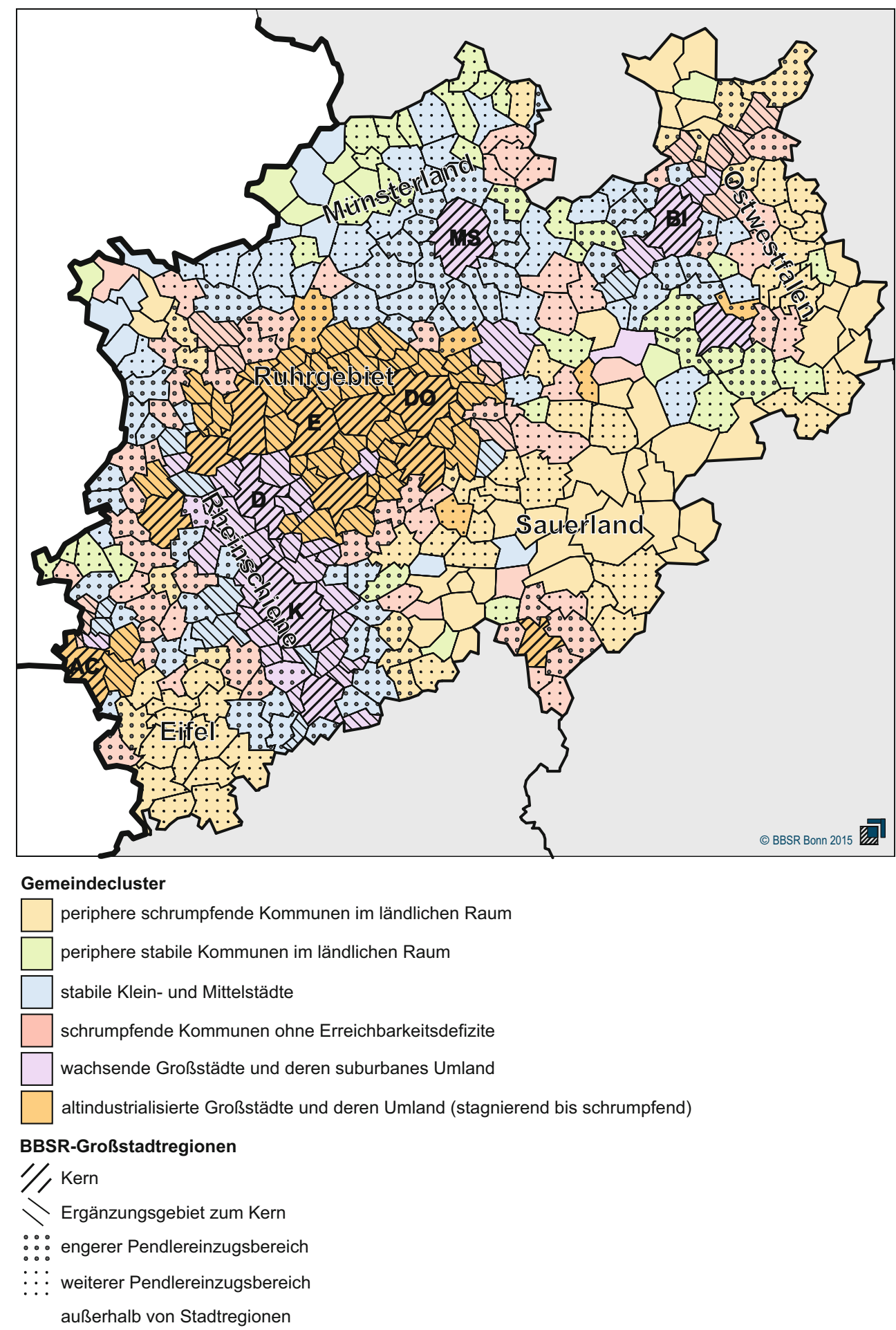

hingegen in einem anhaltenden Prozess des wirtschaftlichen Strukturwandels mit rückläufigen Bevölkerungszahlen. Im Allgemeinen ist der Anteil an Kindern und Jugendlichen hier gering und diese Gemeinden haben zukünftig mit einer überdurchschnittlichen Sterberate zu rechnen. In diesem Cluster finden sich vor allem die Städte und Gemeinden des Ruhrgebietes und des Bergischen Landes.

\section{Planerische Handlungserfordernisse differenziert nach Clustern}

Welche Handlungserfordernisse ergeben sich nun für die Gemeinden in den einzelnen Clustern? An dieser Stelle können und sollen keine auf einzelne Gemeinden oder Gemeindetypen zugeschnittenen Lösungsansätze vorgestellt 
werden. Es werden lediglich den Gemeindeclustern zugeordnete, grundsätzliche Handlungserfordernisse benannt, die die Bandbreite unterschiedlicher Herangehensweisen je nach Betroffenheit verdeutlichen sollen. Über die Eignung und Ausgestaltung bestimmter Maßnahmen muss von den verantwortlichen Akteuren vor Ort im konkreten Fall entschieden werden. Für weiterführende Hinweise zu möglichen Strategien und konkreten Maßnahmen sei auf die in Kapitel 2 dieses Beitrages genannten Veröffentlichungen zum Thema verwiesen.

In Cluster 5 (,wachsende Großstädte und deren suburbanes Umland") sind die geringsten Probleme zu erwarten. In diesem Cluster treffen niedrige Anteile älterer Einund Zweifamilienhäuser am Wohnungsbestand auf eine hohe Wohnraumnachfrage, sodass die älteren Ein- und Zweifamilienhäuser im Zuge eines Generationenwechsels auf den Markt kommen und modernisiert werden. Handlungserfordernisse können sich hier dennoch ergeben, etwa wenn Nachverdichtungs- und Strukturerhaltungsinteressen kollidieren. In solchen Fällen kann eine Moderation oder Mediation zwischen den alteingesessenen Bewohnern und neuen Eigentümern oder Investoren erforderlich sein. Vor dem Hintergrund einer generell hohen Nachfrage nach Ein- und Zweifamilienhäusern in diesem Cluster können Maßnahmen zur Unterstützung eines frühzeitigen Generationenwechsels in den Quartieren sinnvoll sein, wie beispielsweise die Schaffung altengerechten Wohnraums im Quartier. Dies dient insbesondere dazu, die in diesem Cluster bestehende Nachfrage nach Ein- und Zweifamilienhäusern in den Bestand zu lenken und somit den flächenextensiven Neubau in diesem Marktsegment eindämmen zu helfen.

Etwas anders stellt sich die Lage in Cluster 6 (,altindustrialisierte Großstädte und deren Umland“) dar. Auch hier sind die Ein- und Zweifamilienhausbestände unterrepräsentiert, jedoch ist die Lage auf dem Wohnungsmarkt weniger angespannt. Durch fallende Preise für Ein- und Zweifamilienhäuser aus dem Bestand reguliert sich der Wohnungsmarkt selbst. Probleme können das gesellschaftliche $\mathrm{Zu}-$ sammenleben betreffen, beispielsweise wenn sich ehemals homogene Quartiere zunehmend sozial durchmischen oder das Nebeneinander verschiedener Generationen neu erlernt und zum gegenseitigen Vorteil ausbalanciert werden muss. Insgesamt ist das Problemniveau in den Clustern 5 und 6 jedoch so gering, dass sich auf der Grundlage der quantitativen Ergebnisse kein genereller Auftrag für die öffentliche Hand im Umgang mit älteren Ein- und Zweifamilienhausbeständen andeutet.

Cluster 3 und 4 schließen vorrangig Städte und Gemeinden ein, die zwischen den urbanen Kernräumen und den ländlich-peripheren Gebieten gelegen sind. Cluster 3 profitiert von der räumlichen Nähe zu den prosperierenden urbanen Zentren des Landes sowie günstigen demographischen Rahmenbedingungen. Auch in diesem Cluster sind daher derzeit keine Probleme für die Bestandsentwicklung erkennbar. Da die überwiegend vor Inkrafttreten der ersten Wärmeschutzverordnung Ende 1977 errichteten älteren Ein- und Zweifamilienhäuser mit ihrer meist geringen energetischen Qualität in diesem Cluster einen bedeutenden Anteil am Wohnungsbestand ausmachen, sind öffentliche Handlungserfordernisse vor allem in der Unterstützung der energetischen Sanierung sowie - ähnlich wie im Cluster 5 - des Generationenwechsels zu sehen.

In Cluster 4 (,schrumpfende Kommunen ohne Erreichbarkeitsdefizite") befinden sich dagegen Kommunen, die an Stagnations- und Schrumpfungsräume angrenzen. Dieser Cluster ist durch Bevölkerungsverluste und bereits leicht erhöhte Leerstände in älteren Ein- und Zweifamilienhäusern gekennzeichnet. Ungünstige Rahmenbedingungen sowohl innerhalb des Clusters als auch in den angrenzenden Regionen machen negative Entwicklungen wahrscheinlich. Zwar wird durch ein niedriges Preisniveau bislang noch die Marktgängigkeit dieser Häuser gewährleistet, jedoch könnte mittelfristig eine Marktsättigung in diesem Segment erreicht werden, zumal wenn durch weiteren Neubau auf günstigem Bauland der Gesamtbestand an Ein- und Zweifamilienhäusern kontinuierlich wächst. Handlungserfordernisse bestehen hier also in einer Beschränkung des Neubaus und der interkommunalen Kooperation im Baulandmanagement. Anderenfalls besteht die Gefahr, dass eine Vielzahl von Gemeinden mit nur teilweise bebauten Neubaugebieten und zugleich wachsenden Leerständen in Bestandsgebieten zu kämpfen hat. Solche Entwicklungen führen nicht nur zu starken Wertverlusten der betroffenen privaten Immobilien, sondern auch zu Folgekosten und technischen Problemen beim Betrieb kommunaler Infrastrukturen.

Cluster 1 und 2 umfassen die ländlich-peripheren Gebiete Nordrhein-Westfalens und sind von hohen Anteilen der Wohnungen in Ein- und Zweifamilienhäusern am Wohnungsbestand geprägt. Bei den Gemeinden des Clusters 2 (,periphere stabile Kommunen im ländlichen Raum“) zeigen sich keine erhöhten Leerstände im älteren Einfamilienhausbestand. Der Cluster zeigt die Merkmale eines funktionierenden Bestandsmarktes mit rückläufigen Neubauzahlen. Handlungserfordernisse bestehen wiederum lediglich in der bereits erwähnten energetischen Sanierung sowie einer (fortgesetzten) Wohnungspolitik, die auf die Erhaltung und Qualifizierung der Bestände setzt.

Anders sieht es im Cluster 1 (,,periphere schrumpfende Kommunen im ländlichen Raum") aus. Hier gehen nicht nur die Bevölkerungszahlen deutlich zurück, die Gemeinden dieses Clusters zeigen auch bei fast allen anderen Indikatoren - inklusive der Leerstandsquote - die ungünstigsten Ausprägungen und haben somit das höchste Risiko für eine negative Bestandsentwicklung. In diesem Cluster bestehen nicht nur die dringendsten Handlungserfordernisse für die Kommunen, sondern es gilt auch ein Gebot zur in- 
terkommunalen Zusammenarbeit, da die sich abzeichnenden Probleme nicht mehr auf der Ebene einer einzelnen Kommune adäquat angegangen werden können. Stabilisierungsmaßnahmen sollten auf allen räumlichen Ebenen vom einzelnen Objekt bis hin zur Region ansetzen und in einer integrierten Gesamtstrategie koordiniert werden. Bausteine einer solchen Strategie reichen von Vermittlung und Moderation, Maßnahmen zur Verbesserung der energetischen Qualität, über Flächenmanagement und Innenentwicklungsstrategien - auch zur Herstellung einer höheren Dichte, die Nahversorgungsmöglichkeiten verbessern hilft - bis hin zu übergemeindlichen Absprachen und Kooperationen. Kommunen sollten solche Maßnahmen jedoch nicht nach dem Gießkannenprinzip durchführen, sondern frühzeitig räumliche Prioritäten setzen: Denn nicht alle Quartiere in Gemeinden dieses Clusters lassen sich langfristig stabilisieren und infrastukturell absichern.

\section{Fazit}

Nachdem Ein- und Zweifamilienhausgebiete über Jahrzehnte als Selbstläufer galten, ist in den letzten Jahren aufgrund sich wandelnder demographischer und gesellschaftlicher Rahmenbedingungen zunehmend die Frage nach den Herausforderungen und Zukunftschancen älterer Ein- und Zweifamilienhausbestände in quantitativer, qualitativer und raumstruktureller Dimension in den Fokus gerückt. Der vorliegende Beitrag adressiert vor allem die erste und die letzte dieser Dimensionen, indem er die Bandbreite regionaler und kommunaler Betroffenheiten in Nordrhein-Westfalen in den Blick nimmt. Er stellt ein multivariates Indikatoren- und Analysemodell vor, welches auf der Ebene der Kommunen unterschiedliche Betroffenheiten für den Ein- und Zweifamilienhausbestand der 1950er- bis 1970er-Jahre beschreibt. Damit liegt eine Einschätzung zu Marktfähigkeit und Entwicklungsperspektiven älterer Einund Zweifamilienhäuser vor. Grundlage der Analyse sind Indikatoren aus den vier Themenblöcken Demographie, Wohnungsangebot, Raumstruktur und Wohnungsmarkt, die unterschiedliche Einflussfaktoren auf die Entwicklung älterer Ein- und Zweifamilienhausbestände repräsentieren.

Die Two-Step-Clusteranalyse mit dem Likelihood-Maß als Ähnlichkeitsmaß liefert sechs plausible Gruppen. Zu bedenken ist, dass Clusteranalysen als explorative Verfahren nur eine von vielen Lösungen aufzeigen. Die wichtigsten Gütekriterien zur Bewertung sind daher die Interpretationsfähigkeit der Gruppen, die Besetzung sowie die Homogenität innerhalb der Gruppen bei möglichst hoher Heterogenität der Gruppen zueinander. Im Vergleich zum - zumindest in den Raumwissenschaften - häufig angewendeten WardVerfahren trennt das Two-Step-Verfahren die Gruppen mit demographisch wachsenden und schrumpfenden Kommu- nen besser und die Cluster weisen durchschnittlich eine höhere Homogenität auf. Da das Two-Step-Verfahren robust gegen die Annahmeverletzung der Normalverteilung ist und hier eine Bearbeitung oder ein Fallausschluss von AusreiBern oder Extremwerten vor der Clusteranalyse nicht erfolgte, erweist sich das Verfahren als vielversprechende Alternative zum häufig angewendeten Ward-Verfahren. Allerdings gibt es noch kaum raumwissenschaftliche Analysen, die Klassifizierungen mit dem Two-Step-Verfahren nutzen (vgl. Hoops/Schäfer 2008) und die als Erfahrungswerte für eine vergleichende Bewertung des Verfahrens hätten herangezogen werden können. Zur weiteren Validierung des Verfahrens würde eine Übertragung des methodischen Ansatzes auf andere (westdeutsche) Flächenländer beitragen, welche darüber hinaus auch Anhaltspunkte zur Lage älterer Einfamilienhausgebiete in anderen Regionen Deutschlands bieten würde.

Im Ergebnis differenziert die Clusteranalyse sechs Cluster, die unterschiedliche Marktsituationen und somit Entwicklungsperspektiven für dieses Marktsegment beinhalten. Hieraus resultieren auch sehr unterschiedliche Notwendigkeiten des kommunalen Handelns (vgl. Kapitel 5). Planerische Interventionen sind vorrangig in den Clustern notwendig, die durch Schrumpfungsprozesse gekennzeichnet sind. Zunächst einmal verdeutlicht der stark variierende Anteil an Wohnungen in älteren Ein- und Zweifamilienhäusern in diesen Clustern, dass in diesen, trotz vielleicht ähnlicher demographischer und wirtschaftlicher Rahmenbedingungen, unterschiedliche Problemkonstellationen auch unterschiedliche Handlungserfordernisse nach sich ziehen. So ist insbesondere in den häufig stagnierenden oder schrumpfenden altindustrialisierten Großstädten der Anteil der älteren Einund Zweifamilienhäuser so gering, dass diese trotz insgesamt entspanntem Wohnungsmarkt weiterhin Käufer finden (marktgesteuerter Generationenwechsel). Anders sieht dies in den schrumpfenden Regionen aus, in denen die älteren Ein- und Zweifamilienhäuser einen bedeutenden Anteil am Gesamtwohnungsbestand ausmachen. Insbesondere der Cluster 1 (,periphere schrumpfende Gemeinden im ländlichen Raum") lässt aktuell wie zukünftig Vermarktungsprobleme und damit Leerstände verbunden mit einer Unterauslastung der öffentlichen Infrastruktur erwarten. Die räumliche Verteilung dieses Clusters korrespondiert mit bekannten Fallstudien und auch mit den von Dransfeld, Pfeiffer und Platzek (2009: $49 \mathrm{f}$.) antizipierten ,Verlierern' der peripher gelegenen Bestände minderer und mittlerer Qualität. Als weiteren Raumtyp gilt es vorausschauend den Cluster 4 (,schrumpfende Kommunen ohne Erreichbarkeitsdefizite") im Blick zu behalten, da dieser mittelfristig sukzessive in eine ähnliche Problemkonstellation wie Cluster 1 , abrutschen' könnte.

Die diesem Beitrag zugrunde liegenden empirischen Analysen wurden 2014/2015 erarbeitet, sodass in den 
Indikatoren maximal der Zeitraum bis 2012 betrachtet werden konnte. Dementsprechend bleibt der kurzfristige Nachfragedruck am Wohnungsmarkt durch die erhöhte Zuwanderung nach Deutschland unberücksichtigt (vgl. Kapitel 2). Die in diesem Beitrag dargelegten regional unterschiedlichen Betroffenheiten und Handlungserfordernisse im Hinblick auf ältere Ein- und Zweifamilienhausgebiete werden daher zeitlich später eintreten, als auf der Basis der hier analysierten Daten zunächst zu erwarten war. An den Betroffenheiten und Handlungserfordernissen , an sich' ändert sich hingegen nichts, sodass die Kommunen das Handlungsfeld ,ältere Einfamilienhausgebiete“ dennoch vorausschauend beobachten und strategisch angehen sollten.

Die hier dargestellte Clusteranalyse verdeutlicht regional und kommunal unterschiedliche Problemkonstellationen und Betroffenheiten. Sie lässt aber keine Aussagen zu intrakommunalen Entwicklungen zu. Hier besteht weiterer Forschungsbedarf, um fundierte Aussagen treffen können, wo sich intrakommunal zu erwartende Wohnungsüberhänge räumlich niederschlagen werden. Erkenntnisse aus Rheinland-Pfalz deuten an, dass sich Wohnungsleerstände in ländlich peripheren Räumen zunächst eher in (belasteten) Ortskernlagen niederschlagen werden, während Einfamilienhäuser in den 1950er- bis 1970er-Quartieren durch günstigere Lageeigenschaften vorerst noch vermarktbar sind (Ziegler 2013). Über diese intrakommunalen Marktprozesse, z. B. konkurrierende Marktsegmente oder „Filtering-down-Prozesse“, ist bisher wenig bekannt. Solche intrakommunalen Analysen sind insbesondere für den Untersuchungsraum dieses Beitrages geboten, denn die durchschnittliche Einwohnerzahl der nordrhein-westfälischen Gemeinden ist um ein Vielfaches größer als im Bundesschnitt. Die Gemeinden bestehen daher nicht aus einem zusammenhängenden Ort, sondern aus einer Vielzahl an Siedlungsbereichen und Ortsteilen.

Dieser Beitrag verknüpft die sehr unterschiedlichen, teils gegensätzlichen Herausforderungen in den Clustern mit einigen der in der Literatur vorgeschlagenen Handlungsempfehlungen für den Umgang mit älteren Ein- und Zweifamilienhausbeständen. Nicht zuletzt, da entsprechende Maßnahmen bisher nur vereinzelt umgesetzt wurden, fehlt es bis jetzt an einer systematischen Evaluation dieser Maßnahmen bzw. Maßnahmenbündel. Daher bleibt es zukünftigen Untersuchungen in diesem Themenfeld vorbehalten, die praktische Eignung und Angemessenheit solcher Handlungsempfehlungen zu bewerten.

Danksagung Der Beitrag ist im Rahmen der Arbeitsgruppe „Einfamilienhausgebiete der 50er und 60er-Jahre“ der Landesarbeitsgemeinschaft Nordrhein-Westfalen der Akademie für Raumforschung und Landesplanung (ARL) entstanden. Wir möchten den weiteren Mitgliedern dieser Arbeitsgruppe für den fruchtbaren wissenschaftlichen Austausch und insbesondere die zahlreichen Anregungen zu unse- rem Indikatorenkonzept danken. Ein besonderer Dank gilt außerdem Antonia Milbert (BBSR) für die Unterstützung bei der Clusteranalyse.

\section{Literatur}

Adam, B.; Berndgen-Kaiser, A.; Jochimsen, K.; Münter, A.; Zakrzewski, P. (2015): Ein- und Zweifamilienhausbestände der 1950er bis 1970er-Jahre in NRW. Indikatoren zur Einschätzung der Lage. In: Forum Wohnen und Stadtentwicklung 7, 4, 178-184.

Aehnelt, R.; Winkler-Kühlken, B. (2008): Einschätzung der Marktchancen von Reihenhäusern, Einfamilienhäusern und kleinen Mehrfamilienhäusern aus den 1950er und 1960er-Jahren. Berlin. = BBR-Online-Publikation 13/2008. http://www.bbsr. bund.de/BBSR/DE/Veroeffentlichungen/BBSROnline/2008/DL_ ON132008.pdf?_blob=publicationFile\&v $=2$ (21.11.2016).

Aring, J. (2012): Einfamilienhäuser der 1950er bis 1970er-Jahre in Westdeutschland: Eine neue Herausforderung der Stadtentwicklung. In: Eichenlaub, A.; Pristl, T. (Hrsg.): Umbau mit Bestand. Berlin, 69-85.

Aring, J. (2015): Zwischen Traum und Albtraum? Perspektiven älterer Einfamilienhäuser im Umland von Kassel. In: Forum Wohnen und Stadtentwicklung 7, 4, 191-194.

Bacher, J. (1996): Clusteranalyse: Anwendungsorientierte Einführung. München.

Backhaus, K.; Erichson, B.; Plinke, W.; Weiber, R. (2016): Multivariate Analysemethoden. Eine anwendungsorientierte Einführung. Berlin, Heidelberg.

Bahrenberg, G.; Giese, E.; Mevenkamp, N.; Nipper, J. (2008): Statistische Methoden in der Geographie. Band 2: Multivariate Statistik. Berlin, Stuttgart.

Bayerische Staatsregierung (2015): Ältere Einfamilienhausgebiete fit für die Zukunft! Anpassungsstrategien und Empfehlungen für Kommunen. München.

Berndgen-Kaiser, A.; Bläser, K.; Fox-Kämper, R.; Siedentop, S.; Zakrzewski, P. (2014): Demography-driven suburban decline? At the crossroads: mature single-family housing estates in Germany. In: Journal of Urbanism 7, 3, 286-306.

Berndgen-Kaiser, A.; Fox-Kämper, R.; Wiechert, M. (2016): Post-war Single-Family Houses in Europe under Pressure? A Demographic and Economic Framework for the Future Market of Elder SingleFamily Housing Neighbourhoods. In: Articulo - Journal of Urban Research. doi: 10.4000/articulo.3021.

Berndgen-Kaiser, A.; Krajewski, C. (2015): Ältere Ein- und Zweifamilienhausgebiete in Westdeutschland im Umbruch. Status quo, Probleme und Herausforderungen. In: Forum Wohnen und Stadtentwicklung 7, 4, 171-177.

BIB - Bundesinstitut für Bevölkerungsforschung (2015): Wanderungen über die Gemeindegrenzen. http://www.bibdemografie.de/SharedDocs/Bilder/DE/Grafik_des_Monats/ 2012_10_wanderungen_gemeindegrenzen.html (17.11.2016).

Brack, N.; Metzmacher, M. (2009): Wohnungsleerstand. http://www.bbsr.bund.de/BBSR/DE/WohnenImmobilien/ Immobilienmarktbeobachtung/ProjekteFachbeitraege/ Wohnungsleerstand/wohnungsleerstand.html (17.11.2016).

Cunningham-Sabot, E.; Fol, S. (2009): Shrinking Cities in France and Great Britain: A Silent Process? In: Pallagst, K.; Aber, J.; Audirac, I.; Cunningham-Sabot, E.; Fol, S.; Martinez-Fernandez, C.; Moraes, S.; Mulligan, H.; Vargas-Hernandez, J.; Wiechmann, T.; Wu, T. (Hrsg.): The Future of Shrinking Cities - Problems, Patterns and Strategies of Urban Transformation in a Global Context. Berkeley, 17-27.

De Temple, N. (2006): In die Jahre gekommen. Einfamilienhaussiedlungen im soziodemografischen Wandel. In: Die alte Stadt 33, 2, 123-138.

Dransfeld, E.; Pfeiffer, P.; Platzek, C. (2009): Boden 2030 - Anforderungen an die kommunale Bodenpolitik und den Umgang mit Ein- 
familienhausgebieten der 1950er, 1960er und 1970er-Jahre. Dortmund.

Eurostat (2016): Wohnstatistiken. http://ec.europa.eu/eurostat/statisticsexplained/index.php/Housing_statistics/de (16.11.2016).

Fina, S.; Planinsek, S.; Zakrzewski, P. (2010): Suburban crisis? Demand for single family homes in the face of demographic change. In: Europa Regional 17 (2009), 1, 2-14.

Gatzweiler, H.-P. (1996): Ziele und Wege kleinräumiger Bevölkerungsprognosen: Zusammenfassende Wertung der Diskussion. In: ARL - Akademie für Raumforschung und Landesplanung (Hrsg.): Methodische Ansätze kleinräumig differenzierender Bevölkerungsfortrechnungen. Hannover, 4-13. = Beiträge der ARL 132.

Haase, A.; Bernt, M.; Großmann, K.; Mykhnenko, V.; Rink, D. (2016): Varieties of shrinkage in European cities. In: European Urban and Regional Studies 23, 1, 86-102.

Hahne, U. (2010): Wertverlust und Eigenheim - Motivation und Ortsbindung. In: Forum Wohnen und Stadtentwicklung 2, 1, 13-17.

Held, T.; Waltersbacher, M. (2015): Wohnungsmarktprognose 2030. Bonn. = BBSR-Analysen Kompakt 7.

Hohn, U.; Utku, Y. (2015): Wohnquartiere für morgen? Zehn Thesen zur Zukunft von Einfamilienhausgebieten der 1950er bis 1970erJahre. In: Forum Wohnen und Stadtentwicklung 7, 4, 185-188.

Hoops, C.; Schäfer, J. (2008): Clusteranalyse zur Identifizierung von verschiedenen Gruppen von Internetnutzern. In: Statistische Analysen und Studien Nordrhein-Westfalen 51, 17-23.

IBM SPSS (2012): IBM SPSS Statistics Base 21. ftp://public.dhe. $\mathrm{ibm.com/software/analytics/spss/documentation/statistics/21.0/}$ de/client/Manuals/IBM_SPSS_Statistics_Base.pdf (17.11.2016).

IÖR - Leibniz-Institut für ökologische Raumentwicklung (2015): Homes-uP - Single-family homes under pressure? https://www. ioer.de/homes-up (16.11.2016).

IT.NRW (2015): Vorausberechnung der Bevölkerung in den kreisfreien Städten und Kreisen Nordrhein-Westfalens 2014 bis 2040/2060. Düsseldorf.

Krajewski, C. (2015): Was wird aus den Traumhäusern? Einfamilienhausgebiete in Altena im Umbruch. In: Forum Wohnen und Stadtentwicklung 7, 4, 189-190.

Lerbs, O. W. (2014): House prices, housing development costs, and the supply of new single-family housing in German countries and cities. In: Journal of Property Research 31, 3, 183-210.

Maaß, S. (2015): Hier hat Ihre Immobilie auch in 15 Jahren noch Wert. In: Die Welt vom 3. Juli 2015. http://www.welt.de/finanzen/ immobilien/article143510779/Hier-hat-Ihre-Immobilie-auch-in15-Jahren-noch-Wert.html (16.11.2016).

Martinez-Fernandez, C.; Audirac, I.; Fol, S.; Cunningham-Sabot, E. (2012): Shrinking Cities. Urban Challenges of Globalization. In: International Journal of Urban and Regional Research 36, 2, $213-$ 225 .

Müller-Herbers, S.; Skorka, M. (2016): Zwischen altmodisch, begehrt und neuen Entwicklungschancen - Anpassungsstrategien für ältere Einfamilienhausgebiete. In: Informationen zur Raumentwicklung 3, 303-316.

o. V. (2015): Stadtbewohner rücken zusammen. In: Sächsische Zeitung vom 8. Dezember 2015. http://www.sz-online.de/nachrichten/ stadtbewohner-ruecken-zusammen-3270009.html (17.11.2016).

Postlep, R.-D. (2015): Räumliche Aspekte des aktuellen Flüchtlingszustroms. In: Nachrichten - Magazin der ARL 45, 3, 4-5.
Reicher, C.; Hoffschröer, H.; Mecklenbrauck, I.; Polivka, J.; Tries, D.; Hesse, M. (2013): Suburbaner Raum im Lebenszyklus. Berlin. = BMVBS-Online-Publikation 24/2013. http://www.bbsr.bund. de/BBSR/DE/Veroeffentlichungen/BMVBS/Online/2013/DL_ ON24013.pdf? blob=publicationFile \& $v=2$ (16.11.2016).

Rosenbohm, T. (2005): Perspektiven peripher gelegener Einfamilienhausgebiete. In: RaumPlanung 126/127, 120-124.

Schöfl, G.; Schluchter, S.; Schöfl, M.; Speidel, S.; Rist, H.; Iwanow, I.; Eichhorn, D.; Glaser, W.; Glaser, M. (2005): Pilotstudie zur nachhaltigen Entwicklung von Nachkriegssiedlungen (1945-1965) unter besonderer Berücksichtigung von Flächenökonomie und Flächenmanagement. Forschungsbericht FZKA-BWPLUS-2005. Ludwigsburg, Stuttgart Dresden, Tübingen.

Sousa, S.; Pinho, P. (2013): Planning for Shrinkage: Paradox or Paradigm. In: European Planning Studies 23, 1, 12-32.

Spehl, H. (Hrsg.) (2011): Leerstand von Wohngebäuden in ländlichen Räumen. Beispiele ausgewählter Gemeinden der Länder Hessen, Rheinland-Pfalz und Saarland. Hannover. = E-Paper der ARL 12.

Spellerberg, A.; Spehl, H. (2011): Einleitung. In: Spehl, H. (Hrsg.): Leerstand von Wohngebäuden in ländlichen Räumen. Beispiele ausgewählter Gemeinden der Länder Hessen, Rheinland-Pfalz und Saarland. Hannover, 1-2. = E-Paper der ARL 12.

Standl, H. (2006): Wohnungsmarktprobleme im ländlichen Raum Westdeutschlands. Das Beispiel der Schrumpfungsregion NOBayern. In: Berichte zur deutschen Landeskunde 80, 3, 295-314.

Statistisches Bundesamt (2016): Vorausberechnung Haushalte in Deutschland. https://www.destatis.de/DE/ZahlenFakten/ GesellschaftStaat/Bevoelkerung/HaushalteFamilien/Tabellen/ VorausberechnungHaushalte.html (16.11.2016).

StMUV - Bayerisches Staatsministerium für Umwelt und Verbraucherschutz; StMAS - Bayerisches Staatsministerium für Arbeit und Soziales, Familie und Integration; StMELF - Bayerisches Staatsministerium für Ernährung, Landwirtschaft und Forsten; StMI - Bayerisches Staatsministerium des Innern, für Bau und Verkehr (2014): Modellprojekt Revitalisierung von Einfamilienhausgebieten der 50er bis 70er-Jahre. Abschlussbericht. München (unveröffentlicht).

Von Seckendorff, C.; Müller-Herbers, S.; Skorka, M. (2014): Was passiert mit alten Einfamilienhausgebieten? Siedlungsentwicklung im Bestand. In: Magel, H. (Hrsg.): Verspielen wir Grund und Boden? Bestands- und Flächenentwicklung im Praxistest. Werte - Kriterien - Instrumente. München, 55-59.

Wiechmann, T.; Bontje, M. (2013): Responding to Tough Times: Policy and Planning Strategies in Shrinking Cities. In: European Planning Studies 23, 1, 1-11.

Wüstenrot-Stiftung (Hrsg.) (2012): Zukunft von Einfamilienhausgebieten aus den 1950er bis 1970er-Jahren. Handlungsempfehlungen für eine nachhaltige Nutzung. Ludwigsburg.

Zakrzewski, P. (2011): In der Übergangszone: Alternde Einfamilienhausgebiete zwischen Revitalisierung, Stagnation und Schrumpfung. In: Schnur, O.; Drilling, M. (Hrsg.): Quartiere im demografischen Umbruch. Beiträge aus der Forschungspraxis. Wiesbaden, 47-66.

Ziegler, K. (2013): Leerstandsentwicklung in Rheinland-Pfalz - Situation und planerische Anforderungen in Dörfern. In: Flächenmanagement und Bodenordnung 75, 5, 214-224. 